\title{
WIYN/Hydra Detection of Lithium Depletion in F Stars of the Young Open Cluster M35 and Implications for the Development of the Lithium Gap ${ }^{2}$
}

\author{
Aaron Steinhauer ${ }^{3}$ and Constantine P. Deliyannis \\ Astronomy Department, Indiana University, 727 E. Third St., Bloomington, IN \\ 47405-7105; aarons@astro.ufl.edu,con@astro.indiana.edu
}

\begin{abstract}
We report discovery of significant depletion of $\mathrm{Li}$ on the surfaces of $\mathrm{F}$ dwarf stars in the 150-Myr-old open cluster M35, analagous to a feature in the 700-Myrold Hyades cluster that has been referred to as the "Li gap." We have caught the gap in the act of forming: using high resolution, high $\mathrm{S} / \mathrm{N}, \mathrm{WIYN}^{1} / \mathrm{Hydra}$ observations, we detect Li in all but a few M35 F stars; the maximum depletion lies at least $0.6-0.8$ dex below minimally depleted (or undepleted) stars. The M35 Li depletion region, a) is quite wide, with clear depletion seen from $6000 \mathrm{~K}$ to $6700 \mathrm{~K}$ or hotter; b) shows a significant dispersion in Li abundance at all $T_{\text {eff }}$, even with stars of the same $T_{\text {eff }}$; and c) contains undepleted stars (as well as depleted ones) in the (narrow) classical Hyades gap region, which itself shows no undepleted stars. All of these M35 Li depletion properties support rotationallyinduced slow mixing as the primary physical mechanism that forms the gap, and argues against other proposed mechanisms, particularly diffusion and steady main sequence mass loss. When viewed in the context of the M35 Li depletion properties, the Hyades Li gap may well be wider than is usually recognized.
\end{abstract}

Subject headings: open clusters and associations: individual (M35) — stars: abundances — stars: interiors

\footnotetext{
${ }^{3}$ Current address: University of Florida, Department of Astronomy, 211 Bryant Space Science Center, Gainesville, FL 32611-2055.

${ }^{1}$ The WIYN Observatory is a joint facility of the University of Wisconsin-Madison, Indiana University, Yale University, and the National Optical Astronomy Observatory.

${ }^{2}$ This is paper 21 of the WIYN Open Cluster Study (WOCS).
} 


\section{Introduction}

Abundances of lithium (Li) on the surfaces of stars have proven to be an important diagnostic tool for astronomical investigations ranging from cosmology to stellar structure and evolution. In the stellar interior, $\mathrm{Li}$ is burned in $(\mathrm{p}, \alpha)$ reactions at just a few million degrees, which makes its surface abundance sensitive to physical processes occuring in the outer layers of low mass stars. In the standard theory of stellar evolution (Deliyannis, Demarque, \& Kawaler 1990; Pinsonneault 1997), the only way to affect the surface Li abundance is to convectively mix Li down to regions where it can burn. However, during the main sequence lifetime of $\mathrm{F}$ stars, the surface convection zone (SCZ) occupies only a small fraction (by mass) of the Li preservation region (Deliyannis \& Pinsonneault 1997, hereafter DP97). Thus, subsequent to the age of the Pleiades $(100 \pm 20 \mathrm{Myr})^{3}$, the standard model predicts no Li depletion in F stars. Indeed, F stars in the Pleiades and other young clusters themselves show no significant Li depletion, suggesting that pre-MS Li depletion in these stars, if any, must also have been small. It came as a surprise, therefore, when Boesgaard \& Tripicco (1986) discovered that F stars in the Hyades (700 \pm 50 Myr) had dramatically depleted their surface $\mathrm{Li}$ abundances. It was immediately clear that additional physical mechanisms beyond those usually included in the standard theory were required to explain this phenomenon. Subsequent studies of open cluster Li and Be abundances are revealing a rich variety of stellar Li depletion properties, almost none of which can be accounted for by the standard theory (Deliyannis 2000; Jeffries 2000). It is thus becoming increasingly important to ascertain what physical mechanisms are really operating inside low mass stars, especially since there may be critical implications for interpreting and testing Big Bang nucleosynthesis (Deliyannis, \& Ryan 2004; Pinsonneault et al. 1999) and globular cluster ages (Deliyannis, Demarque, \& Pinsonneault 1989; Deliyannis, Demarque, \& Kawaler 1990; Chaboyer, Demarque, \& Sarajedini 1996).

Three classes of models have emerged as possible explanations for the F dwarf Li gap: 1) diffusion (Michaud 1986), where gravitational settling and thermal diffusion drain Li out of the SCZ and surface, 2) steady main sequence mass loss (Schramm, Steigman, \& Dearborn 1990, "SSD"), where the Li preservation region is simply lost over time (though the required mass loss rates are enormous), and 3) slow mixing driven by instabilities associated with rotation (Pinsonneault, Kawaler, \& Demarque 1990; Pinsonneault, Deliyannis, \& Demarque 1992; Charbonnel et al. 1994, hereafter, C94) and/or gravity waves (Garcia Lopez \& Spruit

\footnotetext{
${ }^{3}$ All cluster ages are derived using Yi et al. (2001) isochrones with $0.2 H_{p}$ convective core overshoot. For discussions on the importance of overshoot see Meynet, Mermilliod, \& Maeder (1993), Yi et al. (2001) and Rosvick \& Vandenberg (1998). Overshoot ages are 25\% larger than ages without overshoot; lithium-test ages are even larger (see Stauffer 2000).
} 
1991; Talon \& Charbonnel 2003). Rotationally-induced mixing could include meridional circulation and related instabilities, and/or instabilities triggered by angular momentum loss. To continue to try to differentiate between these scenarios we pursue here the novel approach of investigating the differences in the predicted morphology of the Li gap in its early (and heretofore uninvestigated) stages. (See $\S 3$ for previously used diagnostics.)

The nearby and only moderately-reddened $(\sim 800 \mathrm{pc}, E(B-V)=0.20)$, very rich open cluster M35 has an ideal age $(160 \pm 20 \mathrm{Myr}$, Deliyannis et al. 2004a) for studying possible early stages of Li gap formation, soon after the age of the Pleiades. There is also excellent membership information from the proper motion study of McNamara \& Sekiguchi (1986, hereafter, MS86) and WOCS radial velocity data (Meibom et al. 2004).

\section{Data and Analysis}

This section provides a brief summary of our methods. For more details see Steinhauer et al. (2004); Steinhauer (2003); and Deliyannis, Steinhauer, \& Jeffries (2002, hereafter, DSJ02).

High S/N spectra of 165 dwarf members (P generally > 0.90 in MS86) of M35 were taken in the region of the $6708 \AA \mathrm{Li}$ I resonance doublet using three configurations of WIYN's Hydra/MOS during three observing runs in 1997 November and December, and 1998 January. Nearly all MS86 members in the range $V=12.25-15$ were observed. Each star was exposed for a total of about 5 hours, yielding S/N per 0.2 Apixel of 100-300 in the co-added spectra. $(\mathrm{R}=18,000=1.9$ pix for the red fibers and $13,000=2.5$ pix for the blue fibers. $)$

Data reduction employed standard IRAF techniques (not dohydra). Fits to the daytime sky spectra were used to correct for the throughput variations between fibers and sky subtraction used an average of $\sim 20$ sky fibers per exposure. Effective temperatures were determined using WOCS BVRI CCD photometry (Deliyannis et al. 2004a). An empirical relation was made between the $B-V$ color of the fiducial single-star sequence and the other colors ( $B-R, B-I, V-R, V-I, R-I)$, and an effective $B-V$ was calculated for each. These six effective $B-V$ 's were then averaged, and this value was converted into $T_{\text {eff }}$ using the $(B-V)-[F e / H]-T_{e f f}$ relation in DSJ02. For an input $[\mathrm{Fe} / \mathrm{H}]$ in that relation, we used the M35 cluster average of $-0.143 \pm 0.014$ (Deliyannis et al. 2004b; Steinhauer 2003, the

error is the standard deviation of the mean, $\sigma_{\mu}$; there may be additional systematic errors) which agrees nicely with the value $-0.21 \pm 0.10$ from Barrado y Navascués, Deliyannis, \& Stauffer (2001, hereafter, BDS01). Then, $\log \mathrm{g}$ is taken from $Y^{2}$ isochrones (Yi et al. 2001), and $\xi$ is determined using Edvardsson et al. (1993). 
The small contribution to the Li equivalent widths, $W(L i)$, from the Fe I line at $6707.45 \AA$ was removed using

$$
W\left(F e I_{6707.45}\right)=(43.2 \times\langle B-V\rangle-17.1) \times 10^{\left([\mathrm{Fe} / \mathrm{H}]-[\mathrm{Fe} / \mathrm{H}]_{\text {Hyades }}\right)},
$$

which was determined empirically from high resolution and very high S/N Hyades Li data of Thorburn, et al. (1993). Absolute Li abundances, $A(L i)^{4}$, were determined by interpolating on the curves of growth of S04.

The error in $\mathrm{W}(\mathrm{Li}), \sigma_{W}$, is derived using the relation from Deliyannis, Pinsonneault, \& Duncan (1993), and depends on the S/N and FWHM of each Li line. Upper limits are simply $3 \sigma_{W}$. The Li line is also sensitive to $T_{e f f}$, but not to $\log \mathrm{g}$ or $\xi$ : in the gap, $\Delta T_{\text {eff }}=100 \mathrm{~K}$ implies $\Delta A(L i)=0.08$ dex. For the WOCS photometry of our stars in a given filter, typical $\sigma_{\mu}$ from multiple measures are less than $0.01 \mathrm{mag}$. Typical internal errors in the $T_{\text {eff }}$, propagated from $\sigma_{\mu}$ of the averaged $B-V$ 's, are 10-50K for known single stars. The reported $\sigma_{A}$ in Table 1 has contributions from $\sigma_{W}$ and $\sigma_{T_{\text {eff }}}$, added in quadrature. Systematic errors due to $E(B-V)$ and the $T_{\text {eff }}$ scale itself may be at least as large as $100 \mathrm{~K}$, but since they would affect the whole ensemble in essentially the same (small) way, they do not affect our conclusions.

Following the initial selection of $P>0.9$ MS86 members, stars were rejected if, a) they are photometric nonmembers in any of our six CMD's, b) they are radial velocity nonmembers from our spectra or from those spectra used in the $V_{\text {rad }}$ study, or c) they appear to be probable binaries based on a photometric and spectroscopic analyses.

\section{Results and Discussion}

Figure 1 shows $A(L i)$ as a function of $T_{\text {eff }}$ for the Pleiades, M35 (this study), and the Hyades. ${ }^{5}$ M35 data from BDS01 are also shown. All data from the literature have been reanalyzed using our methods and photometry, where available, to give the most consistent picture possible. Several aspects of the $\mathrm{Li}-T_{\text {eff }}$ morphology in M35 are worthy of notice.

First, it is clear that, as compared to the nominal upper bound plateau between 6300 and $6800 \mathrm{~K}$ with $\mathrm{A}(\mathrm{Li})=3.2$, many stars between 6000 and $6700 \mathrm{~K}$ (or beyond) have depleted their surface Li abundances. Although a small amount of Li depletion cannot be ruled out for the Pleiades, the Li gap has definitely begun to form in M35. This is clearly illustrated

\footnotetext{
${ }^{4} A(L i) \equiv 12+\log (n(L i) / n(H))$.

${ }^{5}$ See DSJ02 for sources of Pleiades and Hyades data.
} 
in Figure 2, which shows the continuum-normalized spectra in the Li region for four pairs of stars. The stars in each pair have similar $T_{\text {eff }}$, and thus it is not surprising that their Fe I and Ca I line strengths (which depend primarily on $T_{\text {eff }}$ and intrinsic abundance) are identical; the Li abundances, however, are strikingly disparate. (Note that where necessary, spectra of slowly rotating stars were artificially broadened to match their pair.) Although the errors listed in Table 1 are internal (see $\S 2$ ), we stress again that systematic errors due to uncertainties in $E(B-V)$ or $T_{\text {eff }}$ scale do not affect a differential analysis of stars at the same $T_{\text {eff }}$. Formally, the Li differences are significant at the $5-10 \sigma$ level; the depth of the depletion far exceeds our errors. The upper and lower envelope of stars in the gap region of M35 differ by more than 0.5 dex, which corresponds to to a factor of more than 3 in abundance (and in line strength). Thus, M35 illustrates that the Li gap begins to form early.

Second, the Li depletion region is quite wide in Teff. The same four pairs illustrate that its width exceeds $700 \mathrm{~K}$ (from 6000 to $6700 \mathrm{~K}+$ ), which is clearly wider than the canonical Hyades Li gap (the region 6550-6700K that contains the most extreme Hyad depletions). But perhaps this canonical Hyades gap should be viewed as being part of a wider depletion structure: after all, Hyad Li abundances in the region $6300-6550 \mathrm{~K}$ show Li dispersions of about 1 dex and are, themselves, depleted by up to 1 dex or more. Note also the highly overdepleted Hyad at A(Li) 1.5,6000K in Thorburn et al. 1993. Furthermore, it can be argued that although Praesepe has the same age as the Hyades, its Li gap (or, more generally, F dwarf Li depletion region) is also quite wide, with dramatic depletion seen all the way from 5900 to $6700 \mathrm{~K}$ (Soderblom et al. 1993b). And, like the Hyades, Praesepe has a star near $\mathrm{A}(\mathrm{Li}) 1.5,6000 \mathrm{~K}$. It may be the case that wide Li gaps are more typical than the narrower one in the Hyades, even if that one is extended from $6300 \mathrm{~K}$ to $6700 \mathrm{~K}$ as we propose here.

Third, in M35, the full $T_{\text {eff }}$ range from 6000 to $6700 \mathrm{~K}$ shows both undepleted (or minimally depleted) stars as well as depleted ones. By contrast, the Hyades Li gap has no star with $A(L i)>3.0$ in the $T_{\text {eff }}$ range $6350-6650 \mathrm{~K}$, and Praesepe has just a few high-Li stars in its gap. Also, the bottom of the M35 Li depletion region (gap?) seems fairly uniform from 6000 to $6600 \mathrm{~K}$ (though note the two upper limits at $6500 \mathrm{~K}$ ). Does the blue side of the gap typically deplete more precipitously later on, as perhaps suggested by the Hyades, or does significant depletion continue throughout a wider Teff region, as perhaps suggested by Praesepe? Is the Hyades typical or unusual? Data from additional (rich) clusters are needed to address this issue.

All of the results discussed above strongly support models of stellar evolution that include rotationally induced mixing, while arguing against diffusion and mass loss. In the Yale rotational models where mixing is in part driven by angular momentum loss, Li dispersions 
at fixed $T_{\text {eff }}$ are a natural consequence of the fact that stars form with a distribution of initial angular momenta $\left(J_{o}\right)$. Stars with larger $J_{o}$ will eventually lose more angular momentum and deplete more Li. Figure 3 shows the rotational models of DP97 (solid lines) and the diffusion models of Richer \& Michaud (1993, hereafter RM93, dashed lines; both interpolated slightly to an age of $150 \mathrm{Myr}$ ) as compared to our M35 Li data. The general morphology of the wide gap is well-reproduced by the DP97 model predictions, and a reasonable distribution in $J_{o}$ produces exactly the degree of dispersion that is observed. Both minimally depleted and more significantly depleted stars are predicted throughout the gap region. Note the suggestion from the models that the upper bound plateau at $\mathrm{A}(\mathrm{Li})=3.2$ might itself be slightly depleted. This is consistent with the apparently slightly higher upper bound in the Pleiades, although Galactic Li evolution might also create slight differences in the initial cluster $\mathrm{A}(\mathrm{Li})$.

Some predictions of the rotational models of C94 are similar to those of DP97 (see discussion in Deliyannis et al. 1998). Talon \& Charbonnel (1998) improved C94's treatment of angular momentum transport by wind-driven meridional circulation and shear turbulence and concluded that they could explain the hot side $\left(T_{\text {eff }}>6700 \mathrm{~K}\right)$ of the Li gap and other abundance anomalies in more massive stars; however, they overdepleted Li in stars cooler than 6700K. Talon \& Charbonnel (2003) argue that it is plausible that in these cooler stars angular momentum transport is dominated instead by gravity waves (and not magnetic fields), which have less associated mixing (and Li depletion). Note that a distribution in $J_{o}$ still leads to a spread in $\mathrm{A}(\mathrm{Li})$. We call for the construction of detailed models to see whether this interesting approach can reproduce the features of the young M35 Li depletion features discovered here (and of course numerous other $\mathrm{Li} / \mathrm{Be} / \mathrm{B}$ patterns observed in low mass stars).

Diffusion theory has difficulty explaining several aspects of our M35 observations. First, the depth of Li depletion is greater than the predictions of the RM93 diffusion models which, for M35, predict a maximum depth of only 0.3 dex, while we observe depletion of 0.6 dex or more. The more recent diffusion models of Turcotte, Richer, \& Michaud (1998) include complicating effects of turbulence but this tends to dilute the effects of diffusion. The RM93 models also predict a unique $\mathrm{Li}$ abundance at a given $T_{\text {eff }}$, in obvious disagreement with the observations presented here. While one might imagine parameters in the models that could change from star to star to modulate the effects of diffusion, no such parameter has been suggested or explicitly modeled by the studies mentioned above. What is most troublesome for diffusion is the overall morphology of the Li depletion seen in M35. At 150 Myr, the diffusion Li gap of RM93 has its maximum depletion occur at 6900K, and is very narrow, with a half width of about 100K. Our data show significant depletion over more than $700 \mathrm{~K}$, and extending as cool as 6000K. (RM93 also acknowledge that their models don't reproduce 
the Hyades gap very well.) Diffusion cannot be the dominant mechanism forming the Li gap.

Similarly, mass loss cannot be responsible for the depletion seen in Figure 1. In order to strip away the entire Li preservation region of $\mathrm{F}$ stars of the 700 Myr Hyades cluster, SSD report that mass loss rates are required that are more than 10,000 times the current mass loss rate of the Sun. In order to make this proposal viable, they conjectured that pulsationally unstable $\delta$ Scuti stars might be responsible for such high rates, but these stars are only thought to extend as cool as about $6900 \mathrm{~K}$, so the coolest $\delta$ Scuti is hotter than the hottest Li gap star. With the discovery of significant depletion of Li in M35, these mass loss rates must be even higher, and the depletors at $6000 \mathrm{~K}$ cannot possibly be explained by $\delta$ Scuti stars.

It is worth noting that even if not dominant, diffusion may yet play a role in affecting light element abundances of solar-type stars, since it is expected to occur in sufficiently stable radiative layers. Indeed, helioseismology suggests the action of (downward) helium diffusion (Bahcall, Pinsonneault, \& Wasserburg 1995), and the super-Li rich "Li Peak" dwarf J37 of NGC 6633 reveals the action of (upwards) Li diffusion (DSJ02, but see Laws \& Gonzalez 2003). In Fig. 1, just hotter than the $\mathrm{A}(\mathrm{Li})=3.2$ upper bound plateau extending from 6300 to $6800 \mathrm{~K}$, note the four stars with $\mathrm{A}(\mathrm{Li}) \sim 3.3$ between 6900 and $7200 \mathrm{~K}$. It is conceivable that these somewhat high $\mathrm{A}(\mathrm{Li})$ might somehow be related to upwards diffusion; however, the RM93-predicted Peak is not as wide as $300 \mathrm{~K}$, and the Li-depleted stars in the same $T_{\text {eff }}$ range would require a different explanation. Finally, although it is becoming increasingly clear that slow mixing affects the radiative layers below the SCZs of low mass stars, the challenge remains to decipher what combination of specific physical mechanisms are at work.

We conclude that the study of the timing and morphology of the formation of of the $\mathrm{Li}$ gap provides a powerful new method to study the physical mechanisms occuring inside stars. Results from applying this method to M35 join a variety of other evidence that points to the action of slow mixing in low mass stars, including the Li-Be depletion correlation (Deliyannis et al. 1998), higher Li in short period tidally locked binaries ("SPTLBs", Thorburn, et al. 1993; Deliyannis et al. 1994; Ryan \& Deliyannis 1995), moderately rapid Li depletion in M67 subgiants (Deliyannis, King, \& Boesgaard 1997; Sills \& Deliyannis 2000), the existence of a moderate Be Gap (Boesgaard \& King 2002) though (so far) no B Gap (Boesgaard et al. 1998), the continued depletion of Li during the main sequence (Jeffries 1997; Deliyannis 2000; Jeffries et al. 2002), and the Li dispersions at fixed $T_{\text {eff }}$ (Thorburn et al.; Soderblom et al. 1993b).

This work has been supported by the National Science Foundation under Grants AST9812735 and AST-0206202. 


\section{REFERENCES}

Bahcall, J. N., Pinsonneault, M. H., \& Wasserburg, G. J. 1995, Rev Mod Phys, 67, 781

Barrado y Navascués, D., Deliyannis, C. P., \& Stauffer, J. R. 2001, ApJ, 549, 452 (BDS01)

Boesgaard, A. M. \& Tripicco, M. J. 1986, ApJ, 302, L49

Boesgaard, A. M., Deliyannis, C. P., Stephens, A., \& Lambert, D. L. 1998, ApJ, 492, 727

Boesgaard, A. M. \& King, J. R. 2002, ApJ, 565, 587

Chaboyer, B., Demarque, P., \& Sarajedini, A. 1996, ApJ, 459, 558

Charbonnel, C., Vauclair, S., Maeder, A., Meynet, G., \& Schaller, G. 1994, A\&A, 283, 155 (C94)

Deliyannis, C. P., Demarque, P., \& Pinsonneault, M. H. 1989, ApJ, 347, L73

Deliyannis, C. P., Demarque, P., \& Kawaler, S. D. 1990, ApJS, 73, 21

Deliyannis, C. P., Pinsonneault, M. H., \& Duncan, D. K. 1993, ApJ, 414, 740

Deliyannis, C. P., King, J. R., Boesgaard, A. M., \& Ryan, S. G. 1994, ApJ, 434, L71

Deliyannis, C. P., King, J. R., \& Boesgaard, A. M. 1997, ASSL Vol. 212: Wide-field spectroscopy, 201

Deliyannis, C. P. \& Pinsonneault, M. H. 1997, ApJ, 488, 836 (DP97)

Deliyannis, C. P., Boesgaard, A. M., Stephens, A., King, J. R., Vogt, S. S., \& Keane, M. J. 1998, ApJ, 498, L147

Deliyannis, C. P. 2000, ASPCS 198, 235

Deliyannis, C. P., Steinhauer, A., \& Jeffries, R. D. 2002, ApJ, 577, L39 (DSJ02)

Deliyannis, C. P., et al. 2004, in preparation

Deliyannis, C. P., Steinhauer, A., Margheim, S., \& King, J. R., 2004, in preparation

Deliyannis, C. P., \& Ryan, S. G. 2004, in preparation

Edvardsson, B., Andersen, J., Gustafsson, B., Lambert, D. L., Nissen, P. E., \& Tomkin, J. 1993, A\&A, 275, 101 
Garcia Lopez, R. J. \& Spruit, H. C. 1991, ApJ, 377, 268

Jeffries, R. D. 1997, MNRAS, 292, 177

Jeffries, R. D. 2000, ASPCS 198, 245

Jeffries, R. D., Totten, E. J., Harmer, S., \& Deliyannis, C. P. 2002, MNRAS, 336, 1109

Laws, C. \& Gonzalez, G. 2003, ApJ, 595, 1148

McNamara, B. J. \& Sekiguchi, K. 1986, ApJ, 310, 613 (MS86)

Meibom, S. et al. 2004, in preparation

Meynet, G., Mermilliod, J.-C., \& Maeder, A. 1993, A\&AS, 98, 477

Michaud, G. 1986, ApJ, 302, 650

Pinsonneault, M. H., Kawaler, S. D., \& Demarque, P. 1990, ApJS, 74, 501

Pinsonneault, M. H., Deliyannis, C. P., \& Demarque, P. 1992, ApJS, 78, 179

Pinsonneault, M. 1997, ARA\&A, 35, 557

Pinsonneault, M. H., Walker, T. P., Steigman, G., \& Narayanan, V. K. 1999, ApJ, 527, 180

Richer, J. Michaud, G. 1993, ApJ, 416, 312. (RM93)

Rosvick, J. M. \& Vandenberg, D. A. 1998, AJ, 115, 1516

Ryan, S. G. \& Deliyannis, C. P. 1995, ApJ, 453, 819

Schramm, D. N., Steigman, G., \& Dearborn, D. S. P. 1990, ApJ, 359, L55

Sills, A. \& Deliyannis, C. P. 2000, ApJ, 544, 944

Soderblom, D. R., Jones, B. F., Balachandran, S., Stauffer, J. R., Duncan, D. K., Fedele, S. B., \& Hudon, J. D. 1993, AJ, 106, 1059

Soderblom, D. R., Fedele, S. B., Jones, B. F., Stauffer, J. R., \& Prosser, C. F. 1993, AJ, 106,1080

Stauffer, J. 2000, ASP Conf. Ser. 198: Stellar Clusters and Associations: Convection, Rotation, and Dynamos, 255

Steinhauer, A. 2003, Ph.D. Thesis, Indiana University 
Steinhauer, A. et al. 2004, in preparation

Talon, S. \& Charbonnel, C. 1998, A\&A, 335, 959

Talon, S. \& Charbonnel, C. 2003, A\&A, 405, 1025

Thorburn, J. A., Hobbs, L. M., Deliyannis, C. P., \& Pinsonneault, M. H. 1993, ApJ, 415, 150

Turcotte, S., Richer, J., \& Michaud, G. 1998, ApJ, 504, 559

Yi, S., Demarque, P., Kim, Y., Lee, Y., Ree, C. H., Lejeune, T., \& Barnes, S. 2001, ApJS, 136,417 
Table 1. Comparison Pair Parameters

\begin{tabular}{lcccc}
\hline \hline Star $^{1}$ & $T_{\text {eff }}$ & $\mathrm{S} / \mathrm{N} / \mathrm{pix}$ & $W(L i)$ & $A(L i)$ \\
\hline 149 & $6749 \pm 25$ & 200 & $33 \pm 5$ & $2.85 \pm 0.07$ \\
212 & $6732 \pm 28$ & 200 & $74 \pm 4$ & $3.28 \pm 0.04$ \\
& & & & \\
429 & $6559 \pm 19$ & 170 & $24 \pm 4$ & $2.55 \pm 0.09$ \\
308 & $6525 \pm 22$ & 150 & $84 \pm 5$ & $3.21 \pm 0.04$ \\
& & & & \\
128 & $6380 \pm 47$ & 170 & $32 \pm 6$ & $2.56 \pm 0.10$ \\
$106_{r}$ & $6376 \pm 7$ & 175 & $83 \pm 3$ & $3.08 \pm 0.02$ \\
& & & & \\
313 & $6010 \pm 60$ & 150 & $43 \pm 6$ & $2.42 \pm 0.09$ \\
$192_{r}$ & $5986 \pm 24$ & 95 & $115 \pm 6$ & $2.98 \pm 0.04$ \\
\hline
\end{tabular}

${ }^{1}$ star identifications from McNamara \& Sekiguchi (1986); stars with $r$ designation have been artificially rotationally broadenend in Figure 2 to match their pairs. 


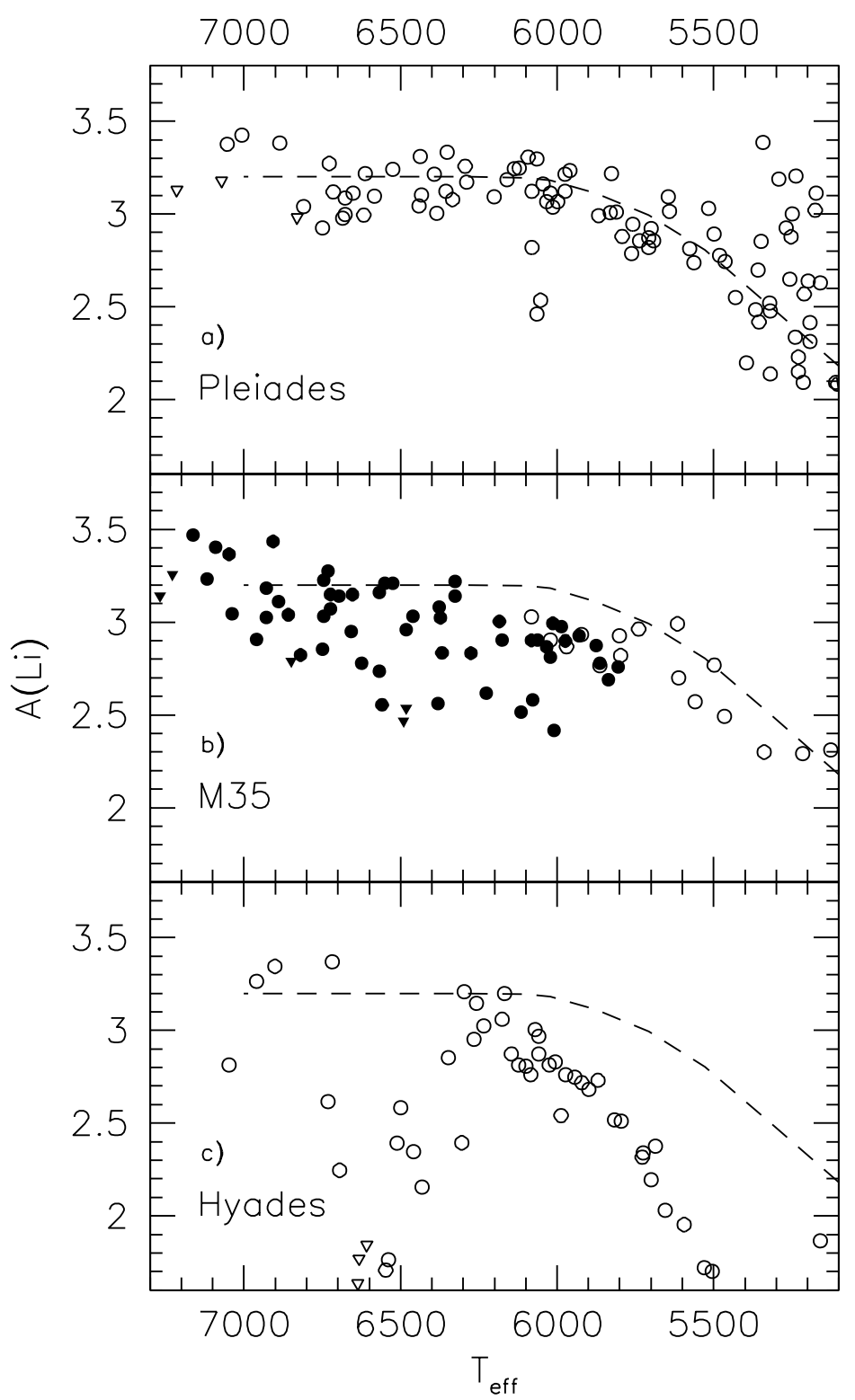

Fig. 1. - Lithium abundances vs. $T_{\text {eff }}$ for probable single stars in (a) the Pleiades, (b) M35, and (c) the Hyades. Solid symbols are results from this work, open symbols are data reanalyzed using methods from this paper. Circles are detections, while triangles denote upper limits. Dashed lines indicate the Pleiades trend. 


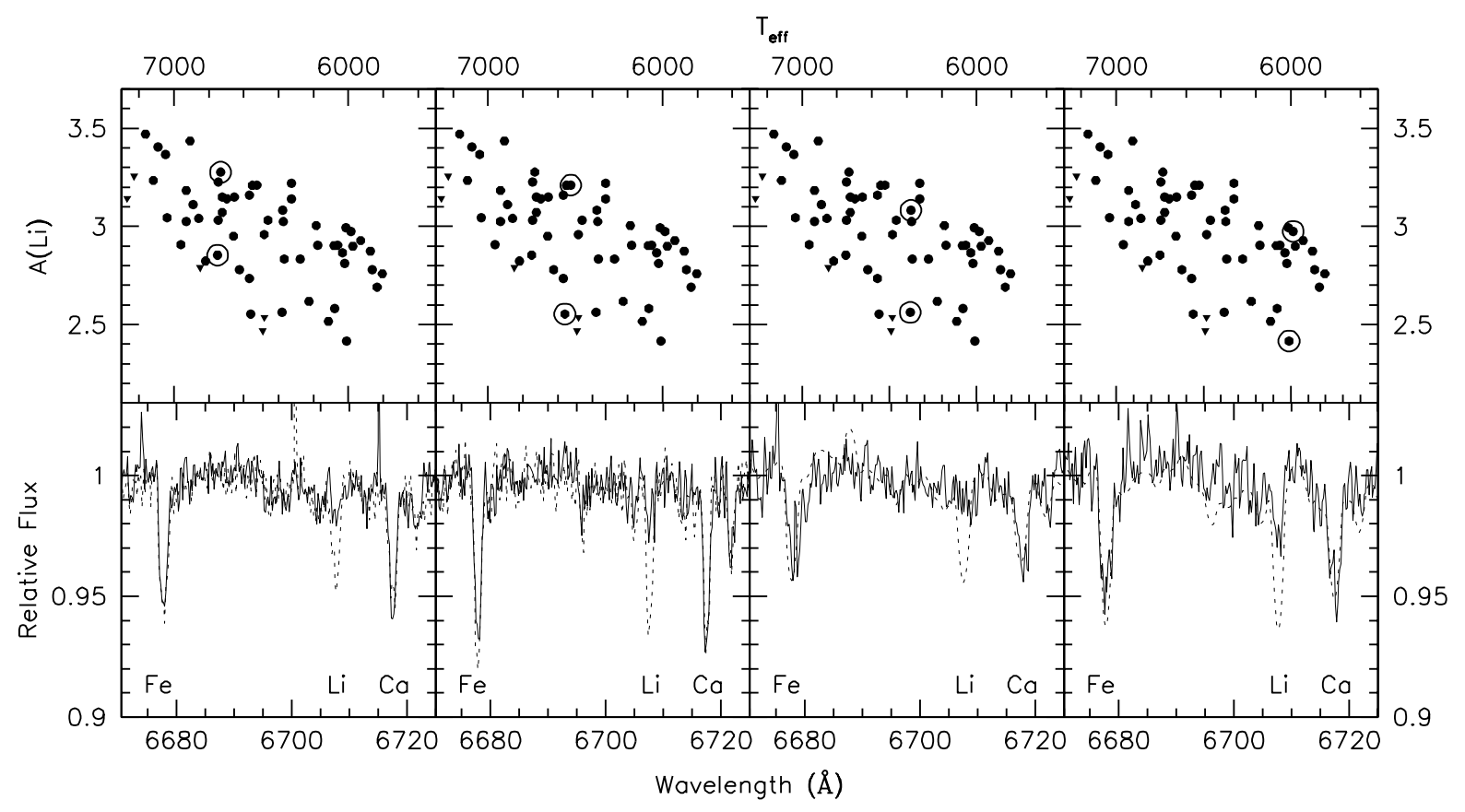

Fig. 2.- Spectra in the Li I $6707.8 \AA$ region for four pairs of M35 dwarfs, of similar $T_{e f f}$, but different $\mathrm{A}(\mathrm{Li})$. Note that some of the spectra have been artificially broadened to match the faster rotator. 


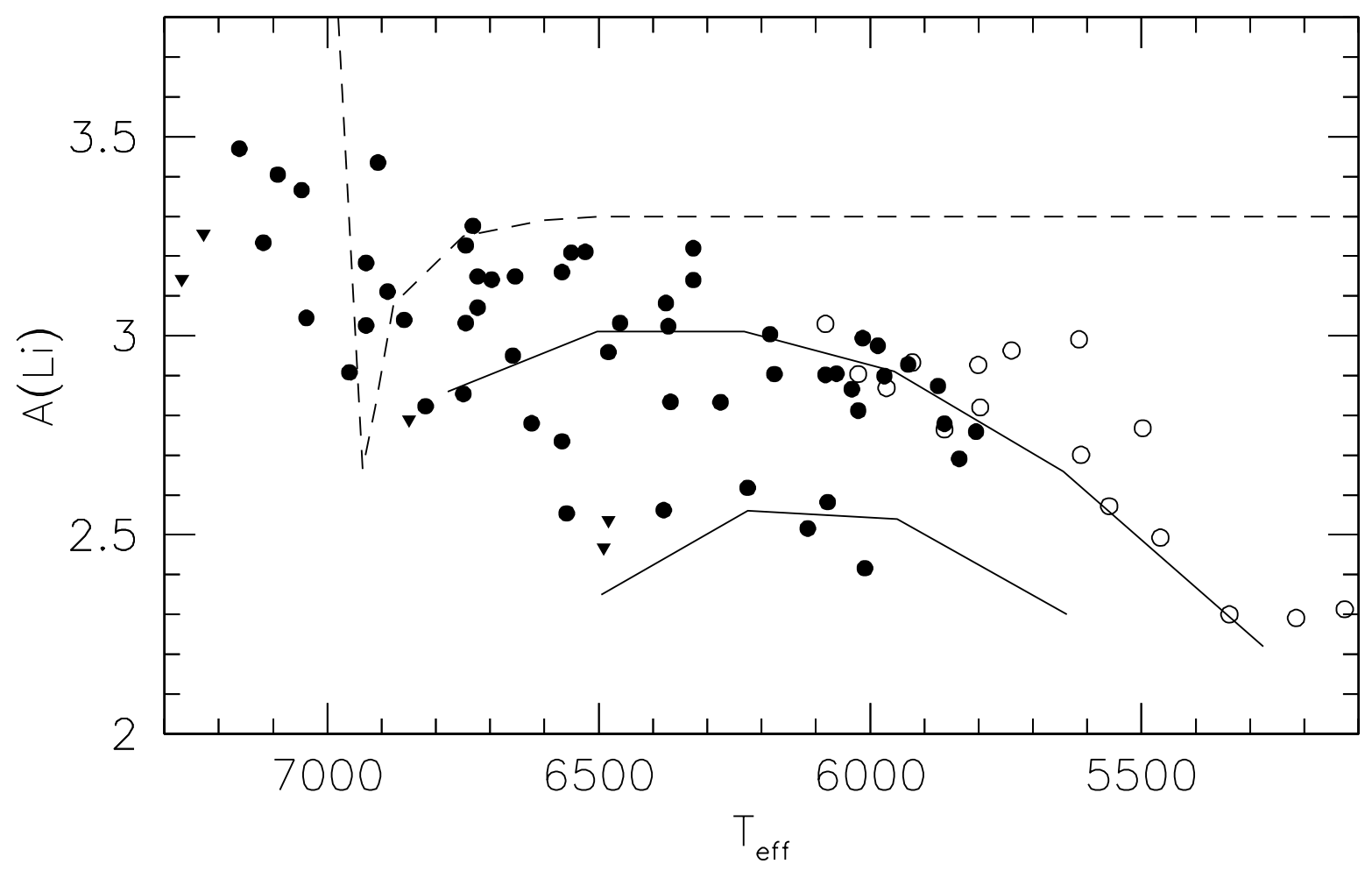

Fig. 3.- Li abundances in M35 with solid symbols from this paper, and open symbols from BDS01 as reanalyzed here. The dashed line represents predictions from the diffusion models of Richer \& Michaud (1993) and the solid lines from the rotationally induced mixing models of Deliyannis \& Pinsonneault (1997), with the upper curve corresponding to $v_{\text {init }}=10 \mathrm{~km} / \mathrm{s}$ and the lower curve to $v_{\text {init }}=30 \mathrm{~km} / \mathrm{s}$. Both models have been interpolated slightly to $150 \mathrm{Myr}$ and assume a meteoritic value of the inital abundance $(A(L i)=3.3)$. 